\title{
A SYNTHETIC PEPTIDE DERIVED FROM TROUT HEPCIDIN INDUCES CYTOKINE EXPRESSIONS IN EUROPEAN SEA BASS (DICENTRARCHUS LABRAX)
}

\author{
Claudio Álvarez ${ }^{1,2,4 *}$, Félix Acosta $^{3 *}$, B. Vega ${ }^{3 *}$, Daniel Montero ${ }^{3 *}$, Fanny Guzmán $^{4 *}$, Elisa \\ Torres $^{1 *}$ and Luis Mercado ${ }^{1, *} \S$ \\ ${ }^{1}$ Grupo de Marcadores Inmunológicos, Laboratorio de Genética e Inmunología Molecular. \\ Instituto de Biología. Facultad de Ciencias, Pontificia Universidad Católica de Valparaíso. \\ ${ }^{2}$ Programa de Doctorado en Biotecnología, Universidad Técnica Federico Santa María/Pontificia \\ Universidad Católica de Valparaíso, Chile. \\ ${ }^{3}$ Grupo de Investigación en Acuicultura, Universidad de Las Palmas de Gran Canaria, España. \\ ${ }^{4}$ Nucleo Biotecnología Curauma, Pontificia Universidad Católica De Valparaíso, Chile.
}

Antimicrobial peptides are destructive against microorganisms and play a role in modulating the immune response, due to which these peptides have also been termed host defense peptides (HDPs). In a previous study, we demonstrated the antimicrobial activity of a synthetic variant of rainbow trout (Oncorhynchus mykiss) hepcidin and the protector effect of this hepcidin in sea bass (Dicentrarchus labrax) challenged with Vibrio anguillarum. The aim of the present study was to analyze the effect of hepcidin on the expression of key cytokines regulating the immune response in fish. The peptide was synthesized, and molecular mass and purity were confirmed. To analyze cytokine modulation by the synthetic peptide, sea bass were intraperitoneally injected with $25 \mu \mathrm{g}$ of the hepcidin variant or a phosphate buffer. At 1, 7, 14 and 21 days post-injection (dpi), head kidney, spleen, and intestinal portions were extracted. The IL-10, IL-1 $\beta$, and TNF $\alpha$ cytokines were quantified through qPCR. The immunomodulatory effect of hepcidin was quantifiable even weeks after application. The evaluated cytokines showed a cyclical tendency of greater then lesser expression, with upregulation of the three cytokines observed in the spleen 1 and $7 \mathrm{dpi}$, in the intestine 3 and $7 \mathrm{dpi}$, and in the head kidney 1, 3, 7, and $14 \mathrm{dpi}$. These differences are likely related to the immunomodulatory role of each cytokine, with the most important being the regulatory role in the head kidney. These observations are probably associated with molecular functions underpinning the immunomodulatory protector effect of hepcidin against pathogens.

KEYWORDS: immune modulation, fish immunology, fish cytokines, fish pathogens

*These authors contributed equally to this work.

$\S_{\text {Corresponding author. Tel.: +56 322274860. E-mail address: luis.mercado@pucv.cl }}$ 